\title{
LA PRESENCIA DE PRODUCTOS COMERCIALES EN LOS MEDIOS DE COMUNICACIÓN Y SU EVOLUCIÓN DE VENTAS: EL CASO DE LA MOTOCICLETA EN ESPAÑA (2001-2010)
}

\author{
Antonio Sanjuán-Pérez: Universidade da Coruña. España \\ Sandra Martínez-Costa: Universidade da Coruña. España \\ Teresa Nozal-Cantarero: Universidade da Coruña. España
}

\section{RESUMEN}

La evolución de un término relacionado con un producto de consumo en las redes sociales, en los medios de comunicación en Internet y en el cine puede crecer de forma paralela a como lo haría su mercado. A su vez éste puede verse influido por el incremento de la conversación sobre él en dichos medios. En ello se fundamentan, entre otras, las teorías relacionadas con los procesos y los efectos mediáticos. El objetivo de este artículo es el de analizar la tendencia del término "motocicleta" tanto en la Red como en el cine y tratar de establecer puntos de relación entre dicha tendencia y la evolución de ventas de motocicletas en nuestro país entre 2001 y 2010. Para ello se empleará una metodología consistente en la recopilación y análisis de una serie de datos, estableciendo unos condicionantes de búsqueda objetiva en Internet, igualdad de términos, cookies, sistemas operativos, resultados por IP, etc.; así como de criterios de selección de películas relacionadas con la "motocicleta". La hipótesis de partida es la de que el aumento del número de películas con una presencia activa de motocicletas representará un incremento de la conversación en la Red, una mayor presencia en las agendas de los medios de comunicación tradicionales y un crecimiento de la evolución de las ventas de motos en España.

PALABRAS CLAVE: Product placement - Agenda - Posicionamiento digital Evolución de ventas

\footnotetext{
${ }^{1}$ Autor correspondiente

Antonio Sanjuán-Pérez: Profesor Titular de Comunicación Audiovisual y Publicidad. Universidade da Coruña. A Coruña. España.
}

Correo: 


\title{
COMERCIAL PRODUCTS PRESENCE IN MASS COMUNICATION MEDIA AND SELLING EVOLUTION: MOTORCYCLE CASE IN SPAIN (2001-2010)
}

\begin{abstract}
The evolution of a term associated with a consumer product on social networking, Internet and film can grow parallel as its market could be. Also it can be influenced by the increase of conversation about in media. They are based on it, among others, the theories concerning the processes and media effects. The aim of this paper is to analyze the trend of "motorcycle" in both network and in the movies, trying to establish points of relationship between that and the evolution of motorcycle's sales in Spain between 2001 and 2010. Methodology consists in the analysis of a data set, establishing some objective conditions in Internet search, equal terms, cookies, operating systems, IP results, etc.., as well as selection's criteria films related to the "motorcycle" term. Hypothesis is that a high number of films with an active presence of motorcycles involve an Internet conversation increase, a greater presence in traditional agenda settings and motorcycle's sales growth in Spain.
\end{abstract}

KEY WORDS: Product placement - agenda setting - digital positioning - sales evolution

\section{INTRODUCCIÓN}

¿Se pueden establecer relaciones entre los términos más buscados en internet y las redes sociales, en la prensa en Red y en el cine y la evolución del mercado de un determinado producto?

Dentro del campo de los procesos y efectos mediáticos este artículo pretende analizar la tendencia de la presencia de los términos "motocicleta" y otros relacionados en los medios de comunicación de masas, junto con la evolución de ventas de motocicletas en España. En concreto, el estudio se centra en el cine y la prensa digital, como medios tradicionales verticales, $\mathrm{y}$ en las redes sociales como fenómeno multiparticipativo.

El método de trabajo sigue el proceso de recopilación de datos, la comparación de los mismos y el estudio de su evolución entre los años 2001 y 2010, teniendo en cuenta las condiciones de macroentorno y las técnicas de búsqueda objetiva. 
En lo relativo al macroentorno se considerarán de manera especial la coyuntura económica y la entrada en vigor de la normativa que permite en España la conducción de motos de hasta 125 cc. con permiso B de circulación. En las técnicas de búsqueda objetiva se establecerán criterios de igualdad de términos, cookies, navegadores, sistemas operativos, IP y resultados por país de origen (en este caso España).

La hipótesis de partida es que un aumento del número de películas con una presencia activa de motocicletas representará un incremento de la conversación en la Red, una mayor presencia en las agendas de los medios de comunicación y un crecimiento de la evolución de las ventas de motos en España.

Este trabajo se enmarca en el contexto de una línea de investigación más amplia sobre la presencia de la moto en los medios de comunicación que lleva a cabo el Grupo de Investigación en Comunicación Audiovisual Interactiva de la Facultad de Ciencias de la Comunicación de la Universidade da Coruña (GRICAI). La finalidad de este artículo es la de contrastar si existe un paralelismo en la evolución de las ventas de motocicletas en España con su presencia en los medios de comunicación.

Para ello se lleva a cabo una investigación de campo sobre el uso del término motocicleta y moto (en inglés y español) en el cine, la prensa digital y las redes sociales. De esta forma se reconoce el papel de integración y difusión que juegan los medios de comunicación de masas, entendiendo que de forma consciente o inconsciente se convierten en modelos de referencia y creadores de estereotipos (Gerbner et alt: 2002, p. 46-67). La exposición continuada a lo largo del tiempo crea además, la tendencia a la estabilización o al cambio en las actitudes del espectador y los medios de comunicación aportan su contribución a la visión del mundo que pueda tener el público (Shanahan \& Morgan: 1999). Estos efectos se complementan también con las interacciones con personas que han utilizado los medios de comunicación (Katz: 1957, p. 61-78).

En una vertiente más comercial la ubicación de marcas en medios como el cine se consideraría como una parte de las comunicaciones integradas de marketing (Kitchen: 2005).

Para la investigación, se propone una metodología de análisis previamente contrastada en estudios sobre la presencia de determinados términos en los medios de comunicación (Cho, Boyle, Keum, \& Shevy, 2008) y sobre la influencia de los mismos en la creación de roles y estereotipos (Mateos de Cabo et alt: 2007). 


\section{METODOLOGÍA}

Este trabajo se articula en torno a cuatro ejes. Por un lado la información sobre la evolución de las ventas de motocicletas en España. Por otro la presencia de motos en el cine nacional e internacional, sus resultados de taquilla y la evolución de dicha presencia respecto a la historia de la producción cinematográfica global. En tercer lugar la aparición del término motocicleta en los periódicos de internet españoles. Y finalmente la presencia de dicho término en la red, medido en su conversación e interés entre el público. Como se puede comprobar a continuación, para obtener los datos necesarios de cada uno de estos cuatro ejes se ha adecuado el término de búsqueda a las fuentes de información correspondientes.

Respecto a los datos de las ventas de motocicletas se analizan los resultados en España en los últimos 10 años (2001-2010) con el fin de identificar las tendencias en su evolución. Dado que en este aspecto influyen factores económicos, políticos, sociales y culturales, se ha tenido en cuenta el análisis de la Asociación Nacional de Empresas de las Dos Ruedas (ANESDOR) y la Dirección General de Tráfico para entender y explicar dichos cambios en el sector.

Para el estudio de la evolución de la presencia de motocicletas en el cine se recurre a IMDB (Internet Movie Database), por ser una de las bases de datos más completas y actualizadas a nivel internacional. Para ello se hace un seguimiento de los términos "motorcycle" y "motorbike" en la línea argumental de las películas catalogadas en dicha base de datos desde 1918 en adelante. El listado de películas se clasifica por años y décadas, como forma de medir su evolución en la producción cinematográfica global. Del total de las películas realizadas en esos años, se detectaron 1291 en las cuales la motocicleta estaba presente.

Los resultados de taquilla de dichas películas en España se obtienen de los datos proporcionados por el Ministerio de Cultura relativos a la década 2001-2010. Respecto a los resultados de taquilla de las películas con motos en el mercado internacional se acude de nuevo a los datos proporcionados por el BoxOffice.

Para el análisis de la presencia de la motocicleta en los medios de comunicación se define la búsqueda entre los periódicos en internet con mayor número de entradas, según el ranking de Alexa. De entre los principales, se escogen tan solo aquellos que tienen versión del periódico en soporte papel, por ser de mayor antigüedad y fiabilidad, además de facilitar así la búsqueda de términos entre los años 2001 y 2010. También se desechan los de tirada gratuita porque su aparición es más reciente y porque algunos de ellos ya no están en circulación. La selección se reduce por lo tanto a las cabeceras "El País", "El Mundo", "ABC" y "La Vanguardia”. Los términos definidos para la búsqueda en estos periódicos en Internet son "motocicleta" y "moto". Del total de los medios de comunicación se contabilizaron 71730 menciones a dichos términos. 
Por último se emplea Google Trends como herramienta de medición de la tendencia en las conversaciones sobre este término en la Red. La búsqueda en este caso se hizo con los términos "motorcycle", "motorbike", "motocicleta" y "moto". Esta última fue hecha acotándola solo a su evolución en España, dado que en otros países puede tener un significado diferente.

Para todas las búsquedas se establecieron criterios de igualdad de términos, se eliminaron las cookies previamente, su buscó en el navegador Safari, bajo sistema operativo Leopard, siempre desde una misma IP y teniendo en cuenta que dichos resultados están condicionados por el país de origen de la búsqueda (en este caso España).

\subsection{Objetivos}

a) Identificar la evolución de la presencia de la motocicleta en el cine entre los años 2001 y 2010.

b) Identificar las tendencias en la presencia de la motocicleta en los medios de comunicación en Internet y de forma especial en el cine.

c) Determinar la relación entre la presencia de motocicletas en el cine y los medios de comunicación de comunicación con la evolución de ventas del vehículo en España entre 2001-2010.

\subsection{Hipótesis}

Para la realización del estudio se tendrán en cuenta las siguientes hipótesis de partida:

H1. El incremento de la presencia de la motocicleta en los medios de comunicación españoles debe coincidir con el incremento de las ventas de motocicletas en España entre 2001 y 2010.

H2. El incremento de las ventas de motocicletas coincidirá con el incremento de la conversación sobre las motos en internet.

H3. Cabe preguntarse si el incremento de la presencia de las motos en el cine coincidirá con el incremento de ventas de motos en España entre 2001 y 2010.

H4. El incremento de películas con presencia de motocicletas coincidirá supuestamente con el aumento de la presencia del término en los medios de comunicación. 


\section{ANÁLISIS Y DISCUSIÓN}

\subsection{La evolución de ventas de motocicletas en España. 2001-2010}

Según datos del Informe del sector de dos ruedas llevado a cabo por Anesdor en 2007, la producción de motos en España se ha duplicado entre 1995 y 2005 (de unos 20 a 40 millones). De 2001 a 2005 se pasó de 25 millones a 40 y sólo entre los años 2004 y 2005 se produce otro incremento de 10 millones.

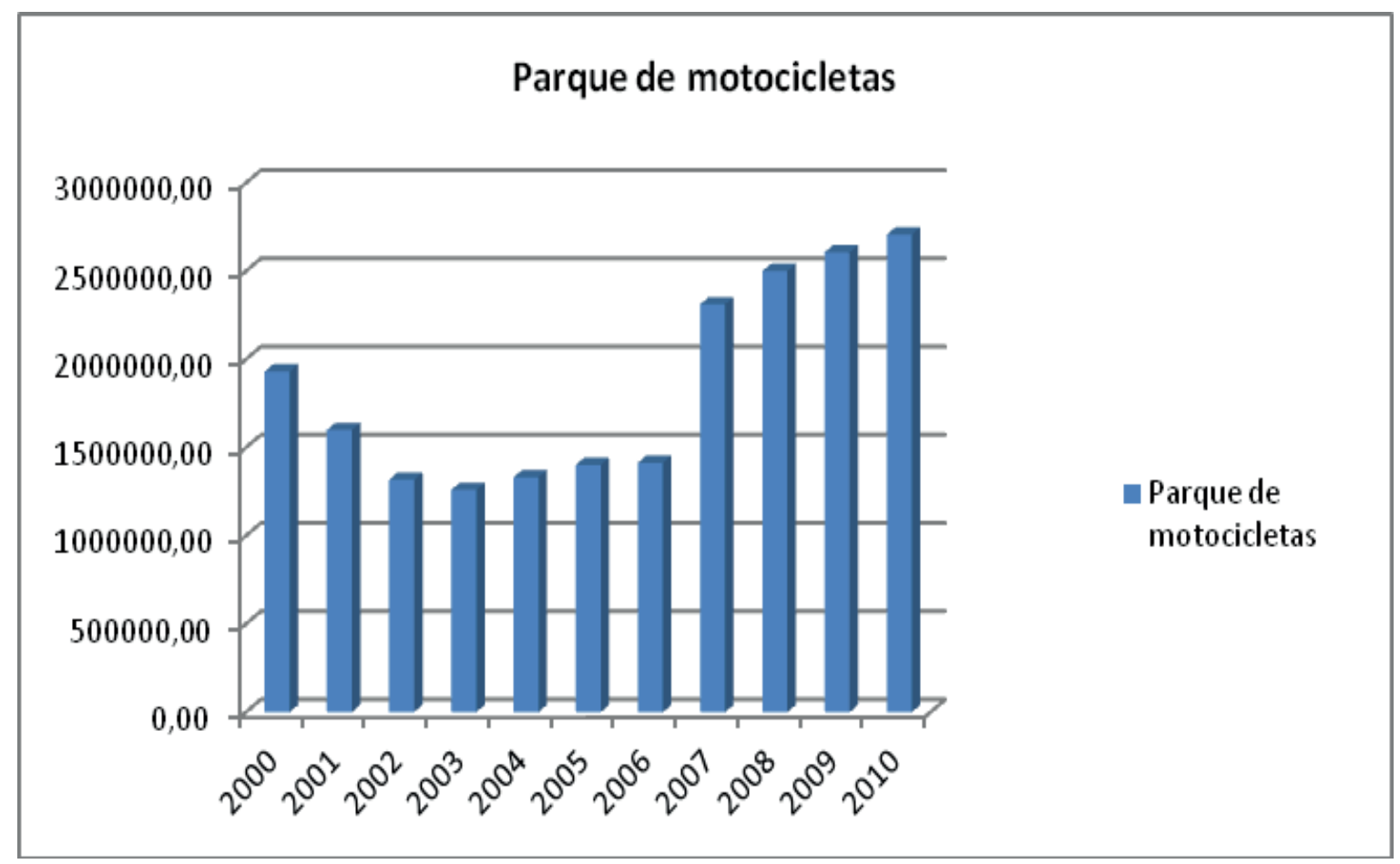

Figura 1. Evolución de ventas en España entre los años 2001-2010. Fuente: Anesdor y Dirección General de Tráfico

La homologación del carnet B con tres años de experiencia y el A1 de motos del 19 de octubre de 2004 supuso un notable y progresivo incremento en las ventas, tal y como se puede ver en el gráfico 1. A pesar de que ese aumento se hace notar a lo largo de 2002, la subida más notable se produce entre 2003 y octubre de 2004. En 2006 España se convierte en el segundo mercado europeo de motos y el tercero de motos y ciclomotores, lo cual implica un claro interés entre el consumidor español por las dos ruedas. 
Evolución 2000-2007 del mercado de vehículos de dos ruedas en los principales paises europeos

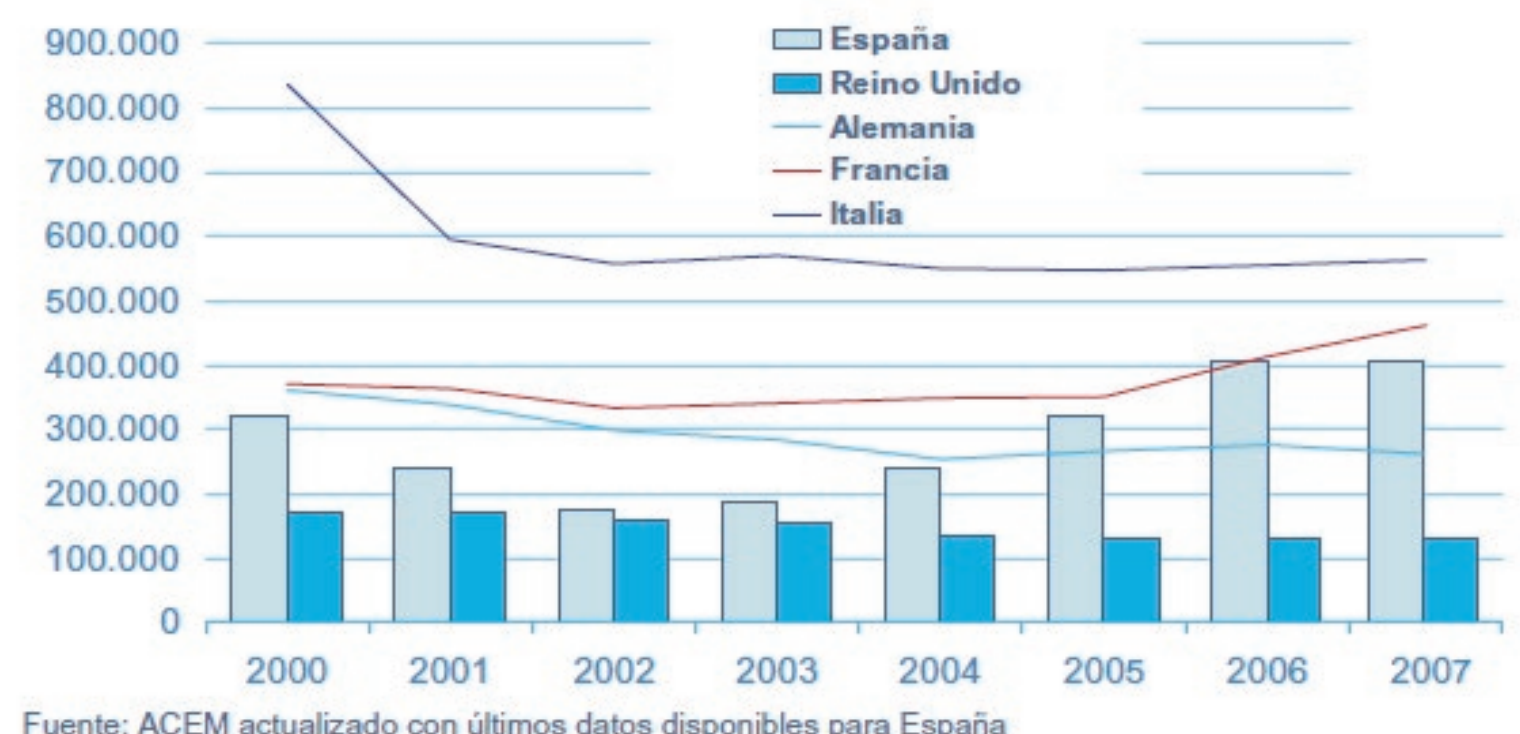

Figura 2. Evolución del mercado de vehículos de dos ruedas en los principales países europeos entre 2001-2007. Fuente: ACEM y ANESDOR

Además, el incremento del parque móvil en nuestro país sigue una tendencia al crecimiento mucho mayor que en la del resto de los de la Unión Europea, tal y como se muestra en la figura 2.

\subsection{La evolución del término "motocicleta" en el cine y los medios de comunicación}

La evolución de la presencia del término "motocicleta" en los medios de comunicación ha ido evolucionando de forma paralela aunque desigual. Cada uno de los cuatro periódicos que se han tenido en cuenta para este estudio vieron incrementado el número de noticias relacionadas con las dos ruedas entre 2001 y 2010, pero de entre los cuatro, destacan "El País" y "ABC" como los de mayor número de noticias relacionadas.

Esta evolución muestra el interés de los medios de comunicación por reflejar la realidad de la moto, al mismo tiempo que el incremento del parque móvil en España supone un aumento de los accidentes de estos vehículos y por lo tanto, una mayor publicación de artículos relacionados. Los cambios en la normativa, las distintas homologaciones para la conducción de motos de gran y pequeña cilindrada y los premios de MotoGP en sus diferentes categorías son factores que condicionan también el número de noticias existentes. 
El estudio de la evolución de estas noticias en los medios de comunicación on line, así como el incremento en las ventas muestra una evolución paralela, tal y como se puede ver en la figura 3, comparativa de la evolución de los medios de comunicación en relación con las ventas de motocicletas en España. Esta gráfica corrobora la hipótesis planteada al comienzo de la investigación como H1.

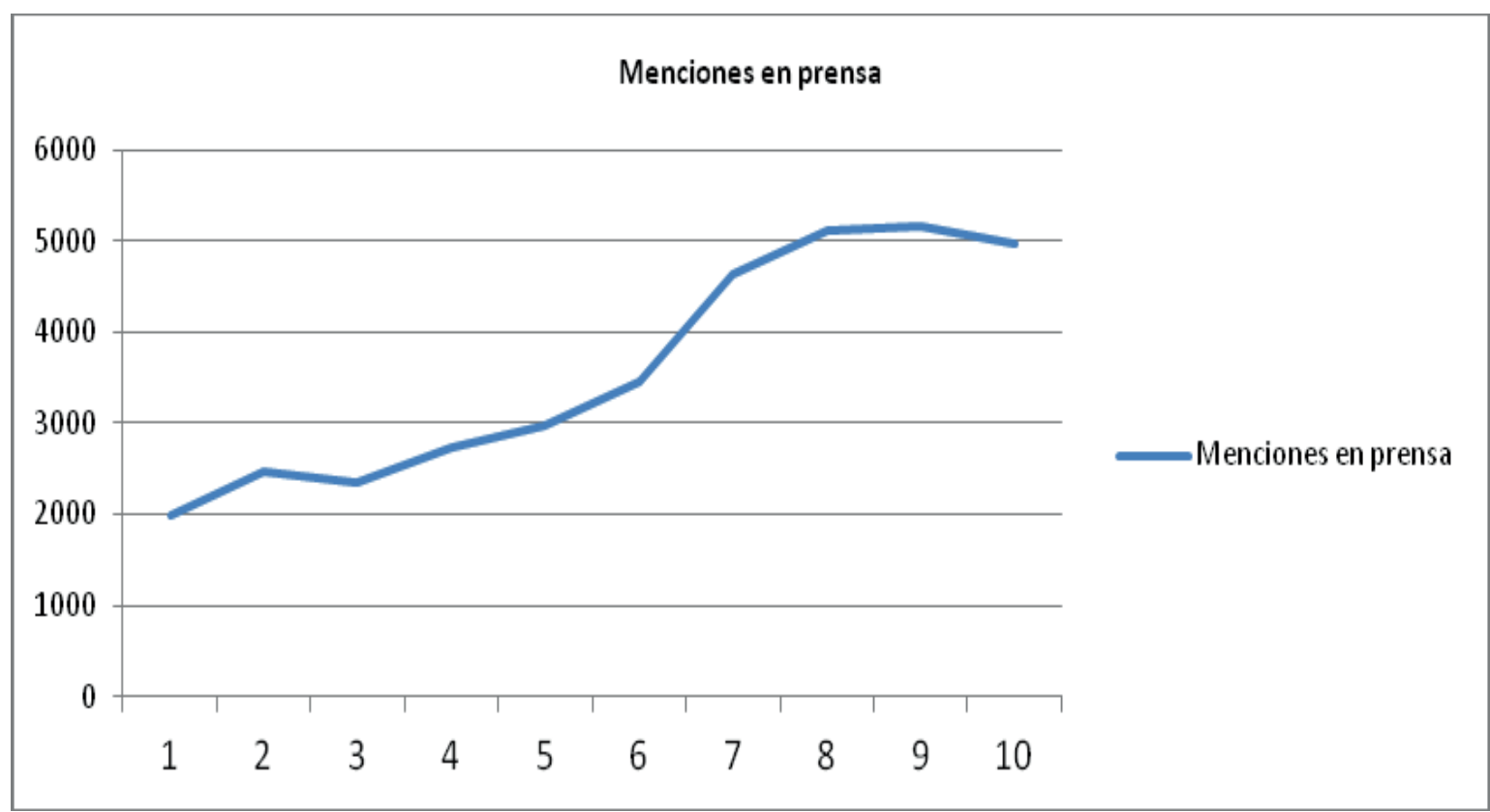

Figura 3. Evolución de las menciones en prensa entre los años 2001-2010. Fuentes. Elaboración propia.

En cambio, el estudio de las tendencias en la conversación sobre los términos "motorcyle" y "motorbike", así como "motocicleta" y "moto" en España indica una evolución también ascendente pero menor.

Para comprobar la correcta evolución en la tendencia de estos términos en la red se llevó a cabo una comparativa con las palabras clave de control "coche" y "bici", por ser igualmente estacional en su uso una de ellas y de utilización constante a lo largo de los años, la otra.

El uso comparativo de estos términos en España arroja los resultados reflejados en la figura 4 y responde a la hipótesis planteada como $\mathrm{H} 2$. 
moto coche bici

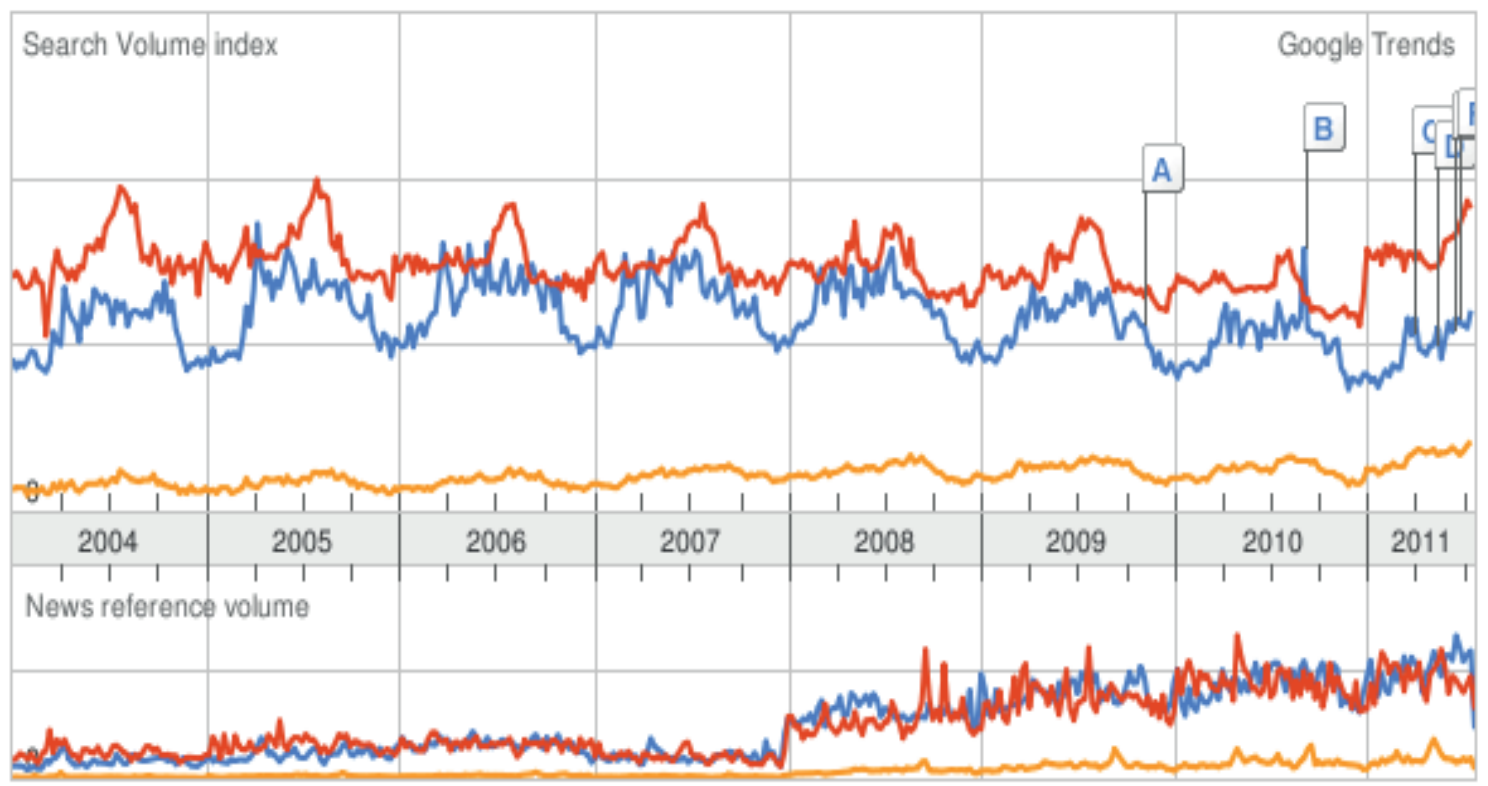

Figura 4. Tendencia de la presencia de los términos "moto", "bici" y "coche" en internet en España entre 2004 y 2010. Fuente. Google Trends.

El estudio mes a mes del término "moto" refleja la estacionalidad en su uso tanto en la conversación en foros como en redes sociales, pero se refleja el notable incremento de las noticias relacionadas en internet. Esa estacionalidad, sin embargo, tal y como se menciona en el informe Anesdor de 2007 es cada vez menor, dada la madurez del mercado y el incremento de motocicletas de gran cilindrada en el parque móvil español.

Por su parte, la producción de películas entre 2001 y 2010 que incluyen en su argumento el término "motocicleta" sufre una evolución ascendente, pero ésta no coincide con su presencia en los medios de comunicación. Tampoco coincide con el incremento de las ventas de motos en España. Los datos indican por lo tanto que no se da la afirmación planteada en las hipótesis H3 y H4. 


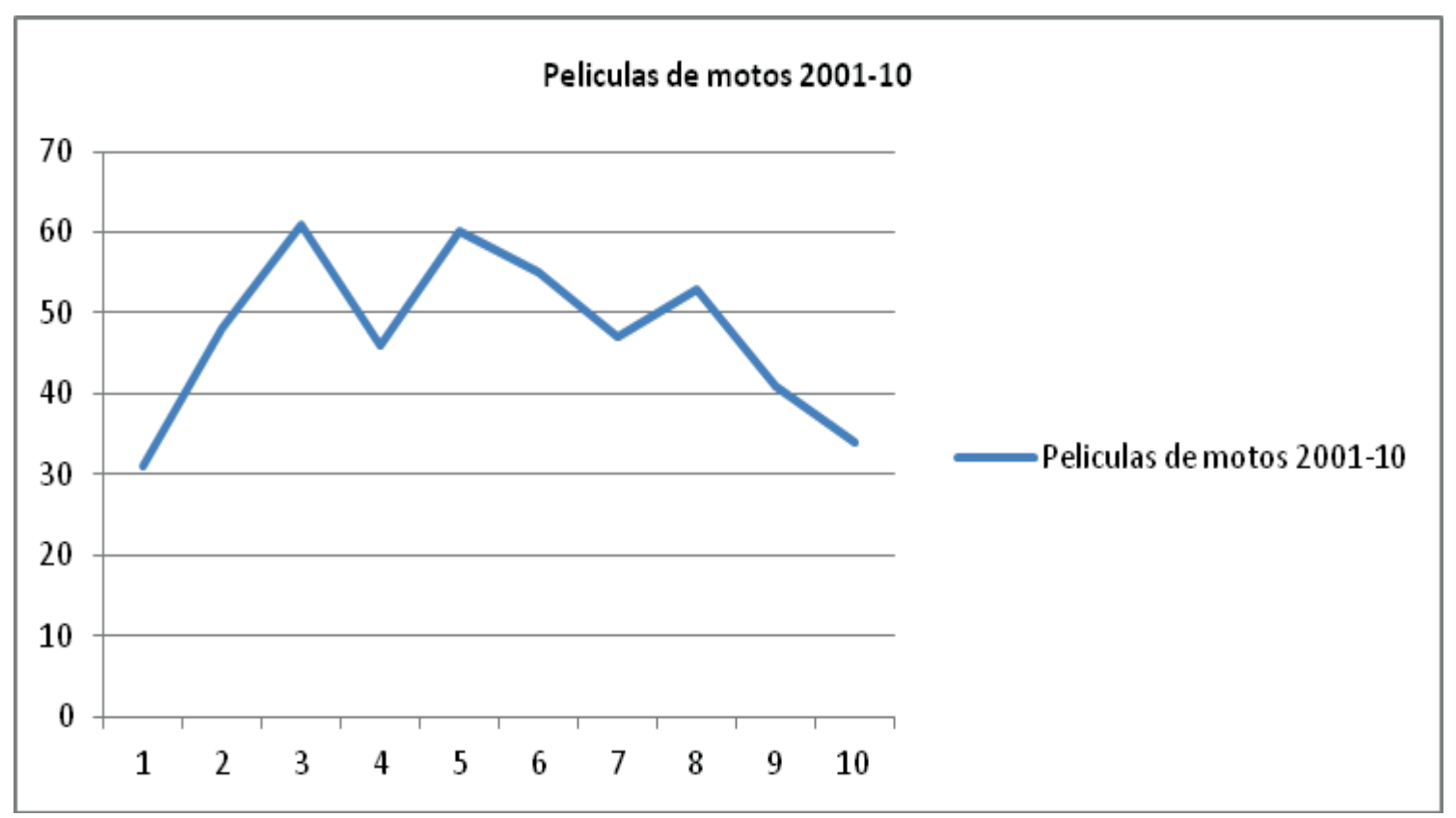

Figura 5. Evolución de las películas con motocicletas entre los años 2001 y 2010. Fuente. Elaboración propia sobre datos de IMDB.

Tal y como se ve reflejado en el gráfico 5, la presencia de la motocicleta en los argumentos de las películas de esa década es irregular, y a pesar de que el final de la década se hace con un incremento en el número de películas, ese incremento es mínimo. La evolución en comparación con la producción mundial de cine refleja, en cambio, que aumenta notablemente el interés por su presencia en el cine.

Tabla 1. Evolución de las películas con motocicletas y de la producción de películas desde la década de 1950. Fuente. Elaboración propia sobre datos de IMDB.

\begin{tabular}{|l|c|c|c|c|c|c|}
\cline { 2 - 7 } & $51-60$ & $61-70$ & $71-80$ & $81-90$ & $91-00$ & 2001-10 \\
\cline { 2 - 7 } & 19.691 & 26.031 & 28.735 & 33.106 & 28.430 & 46.409 \\
$\begin{array}{l}\text { Producción } \\
\text { de películas }\end{array}$ & 32 & 86 & 169 & 194 & 256 & 476 \\
\hline $\begin{array}{l}\text { Películas con los términos } \\
\text { motorcycle y } \\
\text { motorbike en el argumento }\end{array}$ & 32 & & & & \\
\hline
\end{tabular}


El estudio de las películas taquilleras entre los años 2001 y 2010 indica que existe un importante número de ellas que incluyen motocicletas en su argumento.

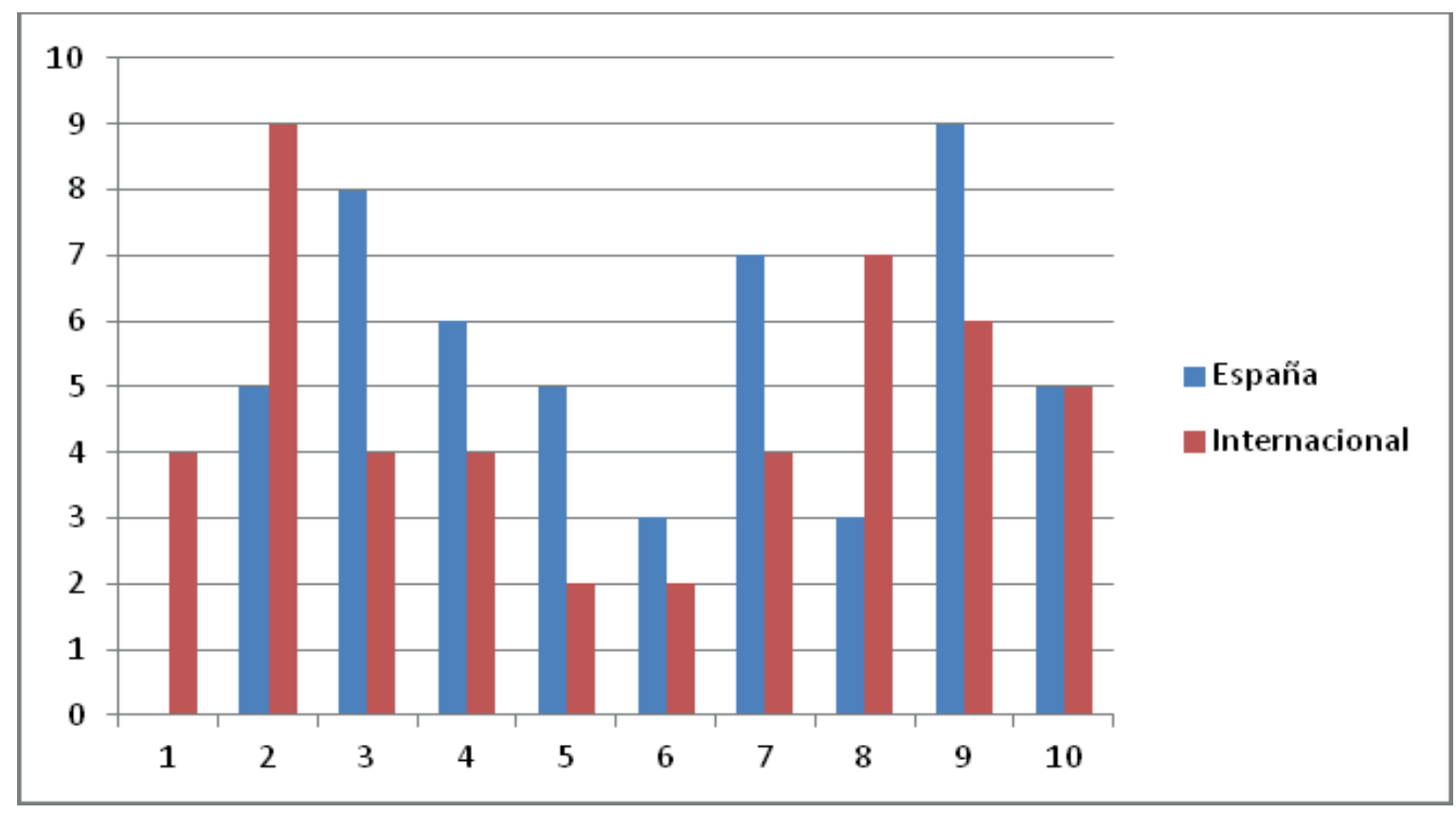

Figura 6. Presencia de películas de motos entre las 25 más taquilleras en España y a nivel internacional. Fuente. Elaboración propia sobre datos de IMDB y del Ministerio de Cultura.

Sin embargo, si hacemos el mismo análisis entre las 25 más taquilleras en España se ve que ese número se incrementa con respecto de la comparativa internacional. Ello se une al aumento del parque móvil de las dos ruedas en nuestro país, mayor que en otros países de la Unión Europea tal y como se mencionaba en el apartado anterior.

\section{CONCLUSIONES}

A modo de conclusión podemos indicar los siguientes puntos:

a) La presencia de la motocicleta en el cine no ha parado de incrementarse en los últimos sesenta años, en términos absolutos y en términos relativos a la producción global de películas.

b) El 38 por ciento de todas las películas con una presencia significativa de motos realizadas en la historia del cine se ha producido en la década 200110.

c) La presencia de la motocicleta en la prensa de información general en España muestra un paralelismo con la evolución de ventas de los vehículos de dos ruedas. 
d) La presencia de la motocicleta en las películas de la primera década del s. XXI no coincide con la evolución de ventas en España.

e) La presencia relativa de películas con motos es superior en España que en el mercado internacional. Los datos de taquilla de nuestro país indican que este tipo de películas tiene una mayor recaudación entre el público español. Las películas que tienen motocicletas son el 1,6\% de la producción mundial, el 5\% de las más taquilleras a nivel mundial y el $10 \%$ de las más taquilleras en España. Esto es indicativo de un alto índice de éxito de este tipo de películas entre el público español.

Por todo ello, cabría inducir que la presencia de motocicletas en el cine y en los medios supone un esfuerzo de comunicación integrada de marketing rentable para el productor de vehículos de dos ruedas. Sin embargo, sería recomendable un estudio de la influencia que tiene esa presencia de la moto, ya que puede no transmitir la imagen más adecuada para inducir al consumidor a su compra y uso. Deberán analizarse por lo tanto, en una segunda etapa, los efectos sociales y la imagen que genera en los medios de comunicación de masas.

\section{REFERENCIAS}

Cho, J., Boyle, M. P., Keum, H. \& Shevy, M. D. (2003). Media, Terrorism, and Emotionality: Emotional Differences in Media Content and Public Reactions to the September 11th Attacks. Journal of Broadcasting E Electronic Media, 47.

Gerbner, G., Gross, L., Morgan, M., Signorelli, N. \& Shanahan, J. (2002). Growing up with television: cultivation processes. En Media Effects: advances in theory and research New Jersey: Lawrence Earlbaum.

Katz, E. (1957). The Two-Step Flow of Communication: An Up-To-Date Report on an Hypothesis. Public Opinion Quaterly, 21: 61-78.

Kitchen, P. \& Burgmann, I. (2004). Integrated Marketing Communication.

Recuperado el 30 de julio de 2011, de

http:/ / onlinelibrary.wiley.com/doi/10.1002/9781444316568.wiem04001/full.

Mateos De Cabo, R., et alt (2007). La presencia de estereotipos en los medios de comunicación: análisis de la prensa digital española. Recuperado el 12/07/2011, de http:/ / bit.ly/qWgzVi.

Shanahan, J. \& Morgan, M. (1999). Television and its Viewers. Cultivation Theory and Research. Cambridge: Cambridge University Press.

VV. AA. (2008). The International Encyclopedia of Communication. Alemania: Wolfgang 


\section{Antonio Sanjuán Pérez}

Doctor en Ciencias de la Información y Profesor Titular de Comunicación Audiovisual desde el año 2003. Entre 1983 y 1998 ejerció el periodismo, entre otros medios, en RNE, La Voz de Galicia, Diario de Galicia y El Ideal Gallego. De 1998 a 2003 trabajó como productor de series documentales. Como investigador ha publicado distintos libros y artículos sobre marketing digital, comunicación de crisis y televisión digital.

\section{Sandra Martínez Costa}

Doctora en Publicidad y Relaciones Públicas y profesora de Comunicación Audiovisual en la Universidad de A Coruña. Sus trabajos de investigación se han centrado sobre todo en el campo del product placement, tema en el que realizó su tesis doctoral y en estudios sobre televisión interactiva y redes sociales.

\section{Teresa Nozal Cantarero}

Doctora en Periodismo con una tesis sobre el cine de Éric Rohmer, y profesora de Comunicación Audiovisual en la Universidad de A Coruña. Sus trabajos de investigación están especializados en el estudio del relato fílmico y el análisis temático en las obras audiovisuales de ficción y no ficción. También ha investigado sobre televisión interactiva. 